\title{
Distortion-free imaging through inhomogeneities by selective spatial filtering
}

\author{
Guy Indebetouw
}

\begin{abstract}
Distortion-free imaging through a system with aberrations is possible for a certain class of input. The input spatial spectrum must be restricted to spatial frequencies which are equally affected by the aberrations (i.e., which experience identical phase shifts $\bmod 2 \pi)$. We demonstrate experimentally that if the aberrations can be localized in the pupil plane, an arbitrary input can be prefiltered to produce a distribution which is imaged without distortion by an aberrant system. Equivalently, the output of the system can be postfiltered to select the information which was imaged without distortion.
\end{abstract}

\section{Introduction}

Imaging through inhomogeneous media or with instruments spoiled by aberrations poses problems of considerable interest in imaging science. Of particular importance is the problem of imaging through dynamically changing disturbances, which requires a real-time adaptive technique for its solution. Over the years, a number of approaches have been proposed and used to solve this problem. The general philosophy has been to acquire some knowledge of the disturbance and use that information to correct the distortion or cancel its effects.

The technique which is proposed in this paper is different. It is based on selection rather than correction. The phase aberrations in the pupil are not being corrected or compensated for. Instead, the pupil is simply blanked out with a mask wherever the phase aberrations depart significantly from a constant phase $\Phi_{0} \bmod 2 \pi$. What is left are those portions of the pupil that act as though they were free from phase errors.

In the next section some of the most successful techniques used for aberration compensation and correction are briefly mentioned and contrasted with the method presented in this paper. The key idea, which is to select and transmit only that part of the input

The author is with Virginia Polytechnic Institute \& State University, Physics Department, Blacksburg, Virginia 24061.

Received 25 January 1990.

0003-6935/90/355262-06\$02.00/0.

(C) 1990 Optical Society of America. information which is imaged integrally by the distorting system, is described and discussed in Sec. III. Its feasibility is demonstrated experimentally in Sec. IV.

\section{Background}

One approach used to compensate for the aberrations of an imaging system consists of recording the distorted output and using knowledge of the transfer function to restore the signal via, e.g., inverse or Wiener filtering. ${ }^{1}$ As is well known, this approach has serious drawbacks and does not lend itself easily to a real-time or adaptive implementation.

Phase conjugation techniques have also been studied extensively as a means of correcting for the phase distortions acquired on transmission through an inhomogeneous medium. Holographic techniques ${ }^{2,3}$ were recently extended to real-time ${ }^{4-6}$ and broadband systems. ${ }^{7}$ Exact phase conjugation, however, requires a double pass through the medium. As a result, the output is located on the same side of the inhomogeneity as the input, which is not a very practical situation. This limitation can be overcome in one-way systems where the distortion is picked up by a carrier wave and phase conjugated before being sent back together with the signal information through the inhomogeneity. ${ }^{8-10}$

An elegant way of imaging through inhomogeneities, which combines superresolution ${ }^{11}$ with phase conjugation to overcome some of the limitations of the latter technique, was recently demonstrated. ${ }^{12}$ The technique relies on the idea that with an extended spatially incoherent illumination, all the spatial frequencies of an input are being transmitted through any one point in the pupil. Imaging through a point pupil is of course insensitive to aberrations, but knowledge of the source is needed to decode the output. The scheme is thus not readily suitable for self-luminous objects. 


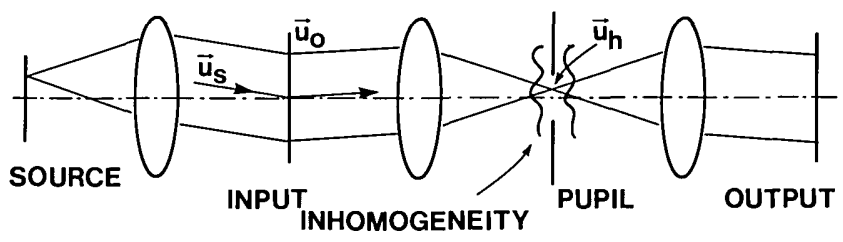

Fig. 1. Generic linear space-invariant imaging system. Each source element illuminates the input with a plane wave of spatial frequency $\mathbf{u}_{s}$, which is scattered to a pupil element $\mathbf{u}_{h}$ by an input spatial frequency $\mathbf{u}_{0}$.

To terminate this short list, mention should be made of the spectacular results obtained recently with adaptive optics, using a deformable mirror for real-time wavefront correction. ${ }^{13}$

As already mentioned, the method described in this paper is selective rather than corrective. The basic idea is extremely simple. An imaging system with aberrations that can be localized in the pupil plane such as shown in Fig. 1 can be represented by a generalized pupil function of the form ${ }^{14}$

$P(\mathbf{u})=A(\mathbf{u}) \exp [i w(\mathbf{u})]$,

where $A(\mathbf{u})$ is the aperture function, $w(\mathbf{u})$ is the phase aberration, and $\mathbf{u}$ is a spatial frequency, which, for a system of focal length $F$ and wavelength $\lambda$, is related to the pupil coordinate via $\mathbf{r}=\lambda F \mathbf{u}$.

Instead of trying to correct the phase distortion $w(\mathbf{u})$, one may decide to restrict the pupil to those areas which have a constant phase $\Phi_{0}$. This can be done with a mask $M(\mathbf{u})$, which transmits only the spatial frequencies $\mathbf{u}_{m}$ for which

$w\left(\mathbf{u}_{m}\right)=\Phi_{0} \bmod 2 \pi$

and blanks out the rest of the pupil.

The solution $\left\{\mathbf{u}_{m}\right\}$ of Eq. (2) is located at the maxima of interference of the wavefront $\exp [i w(\mathbf{u})]$ with a plane wave of uniform phase $\Phi_{0}$. This suggests a simple way of constructing interferometrically the spatial filter which will render the imaging system insensitive to the inhomogeneity. The situation is depicted in Fig. 2. A plane wave (obtained from a single point object) transmitted by the inhomogeneity produces a wavefront $\exp [i w(\mathbf{u})]$ in the pupil. A hard clipped interferogram of this wavefront will select the frequencies $\left\{\mathbf{u}_{m}\right\}$ satisfying Eq. (2) and thus constitutes the needed spatial filter.

Since a pupil mask is being used, the image is in general not perfect. Indeed the quality of the image depends on the structure of the mask, and thus on the aberrations themselves, and varies greatly with the state of spatial coherence of the illumination. These points are discussed further in the next section. Nevertheless, however imperfect, the method has the advantage of being very simple both conceptually and in its realization. It is applicable to illuminations having an arbitrary state of spatial coherence as well as to selfluminous objects, and it lends itself to possible realtime or adaptive implementations.

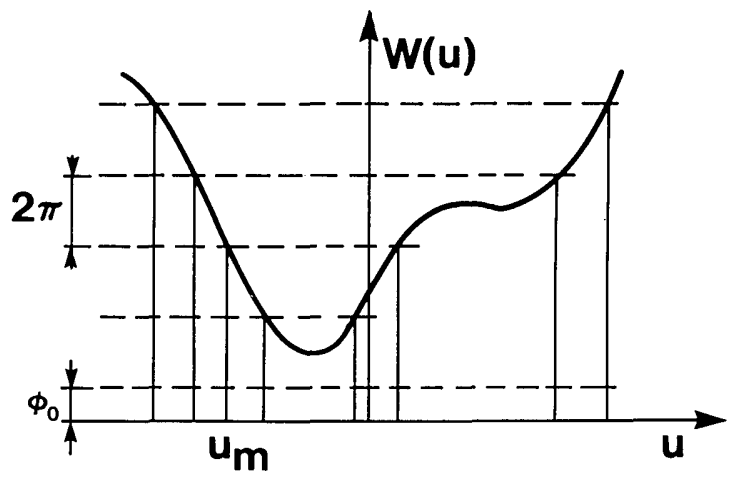

Fig. 2. Interferometric selection of the set of spatial frequencies $\left\{\mathbf{u}_{m}\right\}$, which is identically affected by the inhomogeneity described by a pupil function $w(\mathbf{u})$ (i.e., experiences identical phase shifts $\Phi_{0}$ $\bmod 2 \pi)$.

\section{Discussion of the Method}

Figure 1 shows a generic imaging system with aberrations. For simplicity, a four- $F$ system with unit magnification is depicted. It is trivial to generalize the discussion to an arbitrary imaging system. ${ }^{14}$ With a single point source, the input is illuminated with a plane wave of spatial frequency $\mathbf{u}_{s}=0$, and the imaging is coherent. The source can also be extended, resulting in partially coherent or incoherent imaging. The well known redundancy of an incoherent system is illustrated by the fact that if the source extent is sufficiently large, all the spatial frequencies $\mathbf{u}_{s}$ of the input are being transmitted through any pupil point $\mathbf{u}_{h}$.

With coherent illumination the transfer function of the system is the pupil $P(\mathbf{u})$ itself. An input with an amplitude spatial spectrum $F(\mathbf{u})$ thus leads to an output having a spectrum

$$
G(\mathbf{u})=A(\mathbf{u}) \exp [i w(\mathbf{u})] F(\mathbf{u}) .
$$

In the incoherent case, the optical transfer function (OTF) is the autocorrelation of the pupil, and the spatial spectrum of the output intensity distribution is given by ${ }^{14}$

$$
I(\mathbf{u})=[P(\mathbf{u}) * P(\mathbf{u})] \cdot[F(\mathbf{u}) * F(\mathbf{u})],
$$

where $*$ denotes a correlation product.

A first conclusion that can be drawn from Eqs. (3) and (4) is that, for any aberration function $w(\mathbf{u})$, there exists a class of input which is imaged without distortion by the system. Indeed, if the spatial spectrum of the input amplitude distribution contains only frequencies $\mathbf{u}_{m}$ satisfying Eq. (2), the identity $F(\mathbf{u})$ $\exp [i w(\mathbf{u})]=F(\mathbf{u})$ holds, and the output is identical to the input for any state of coherence.

With an arbitrary input distribution, a pupil mask (or spatial filter) $M(\mathbf{u})$ must be used to transmit only the frequencies satisfying Eq. (2). With this mask, which is designed so that $M(\mathbf{u}) \exp [i w(\mathbf{u})]=M(\mathbf{u})$ $\exp \left(\Phi_{0}\right)$, the output spectra become in the coherent and incoherent cases, respectively,

$$
G_{m}(\mathbf{u})=A(\mathbf{u}) M(\mathbf{u}) \exp \left(\Phi_{0}\right) F(\mathbf{u}),
$$




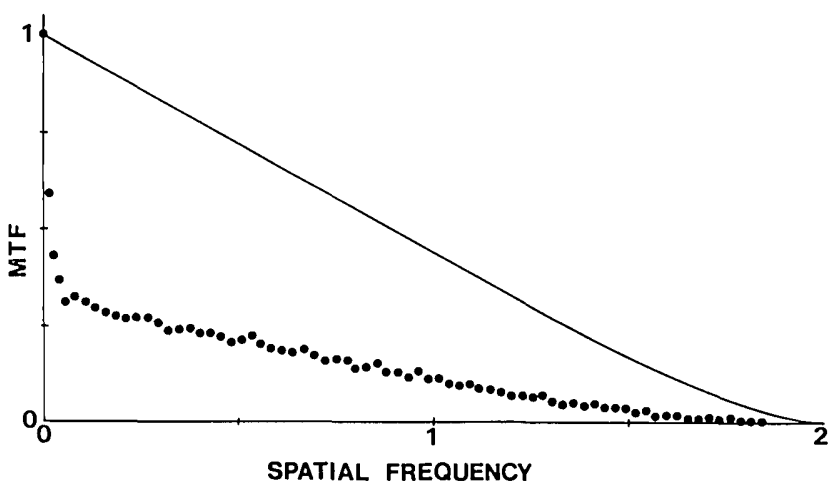

Fig. 3. MTF of the imaging system equipped with the pupil mask shown in Fig. 5 (sliced along a horizontal line).

$$
I_{m}(\mathbf{u})=[A(\mathbf{u}) M(\mathbf{u}) * A(\mathbf{u}) M(\mathbf{u})] \cdot[F(\mathbf{u}) * F(\mathbf{u})] .
$$

It is clear from this result that the quality of the output image depends on the sharpness of the mask, or equivalently, on the level at which the interferogram is being hard clipped. The sharper the mask, the more accurate the selection of the frequencies which are unaffected by the distortion. For example, with coherent imaging, the residual pupil phase error measured in fractions of $2 \pi$ is equal to the sharpness ratio of the hard clipped interferogram (i.e., the ratio of fringe opening to fringe spacing). However, a very sharp mask will transmit little energy, and the output SNR will be consequently reduced. Clearly, an optimum trade-off has to be found in each particular case.

The effectiveness of the method also depends very strongly on the state of coherence of the illumination. In a coherent imaging system, a sharp mask results in a very dilute pupil and drastically changes the coherent transfer function. In general, only a small subset of the spatial frequencies of the input is being transmitted to the output. In principle, however, it is always possible to superimpose sequentially a number of images obtained with different masks, each corresponding to a different phase $\Phi_{0}$. In this way, the entire input information can in principle be transmitted without distortion.

If the imaging system is incoherent, the OTF is determined by the autocorrelation of the pupil. The autocorrelation of a dilute pupil is expected to have a large peak near zero spatial frequency, to fall off more rapidly, and to exhibit more fluctuations than the autocorrelation of a clear aperture. The contrast of the image is thus expected to be lower than that of an image obtained with a clear aperture. However, since the phase of the OTF is uniform, the image is free of the usual distortions, contrast reversals, and artifacts due to a nonuniform OTF phase. Furthermore, as long as the aberrations are sufficiently random, or if the mask contains large closed loop openings (e.g., rings), no spectral information is being lost (although some frequencies may be transmitted with a much reduced contrast).

An example is shown in Fig. 3. For the experiment

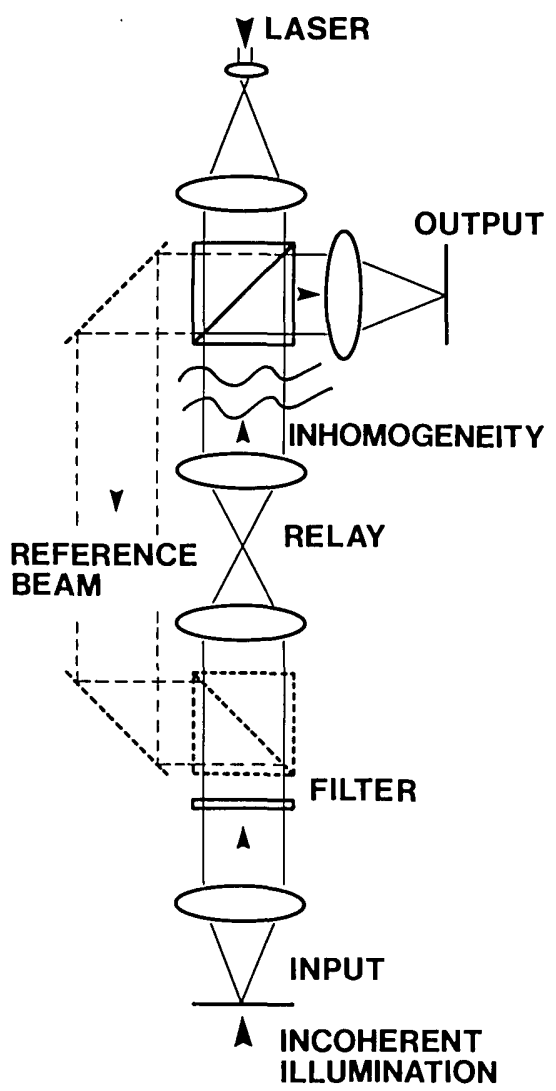

Fig. 4. Experimental system used for the demonstration. The dotted elements and laser beam were used only to record the spatial filter interferometrically.

to be described in the next section, an imaging system was spoiled by using a piece of shower glass near the pupil plane. The mask necessary to make the system insensitive to the aberration was obtained interferometrically. A hard clipped version of the interferogram is shown in Fig. 5. From this interferogram, it is apparent that the aberration function $w(\mathbf{u})$ exhibits several peaks and valleys with a modulation depth of the order of 10 waves. The resulting OTF of the spoiled system is thus expected to not only fall rapidly but to exhibit large phase fluctuations. The MTF of the imaging system equipped with the selective mask of Fig. 5 is shown in Fig. 3. This result was obtained by optical correlation of the mask using a translating template technique. As expected, the MTF has a large central peak leading to low contrast images. However, since the residual OTF phase errors are very small (the mask sharpness ratio is of the order of one-fifth), the image is nearly free of distortion. Furthermore, simple contrast enhancement techniques, based on background reduction, could be used to improve the contrast of the output.

\section{Experimental Results}

The setup of Fig. 4 was used to demonstrate the idea described in the preceding section. To record the filter, a plane wave from a $\mathrm{He}-\mathrm{Ne}$ laser was sent 


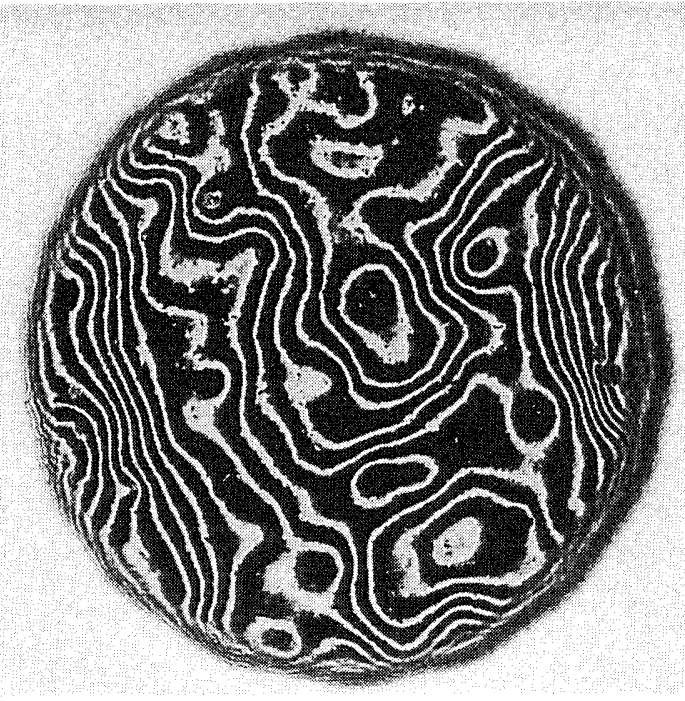

Fig. 5. Interferometrically recorded spatial filter. The inhomogeneity consisted of a piece of shower glass.

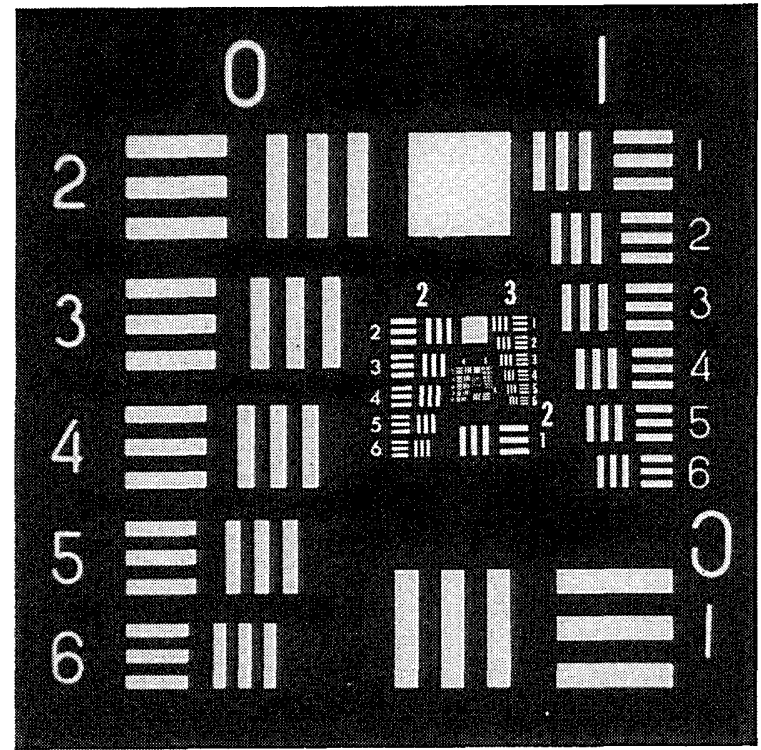

Fig. 6. Unfiltered input imaged without the inhomogeneity (perfect image).

through the inhomogeneity and made to interfere with an unperturbed plane wave. The interferogram was recorded on high contrast film and replaced in its original position. In a first experiment, the incoherently illuminated input was first prefiltered by the recorded spatial filter, then imaged through the inhomogeneity as shown in Fig. 4. Figures 5-9 show the results. The inhomogeneity was a piece of shower glass. Its interferogram produced the filter of Fig. 5. Note that this kind of random pupil phase aberration is typical of imaging systems spoiled by propagation through an inhomogeneous medium such as a turbulent atmosphere. Figure 6 shows the unfiltered input

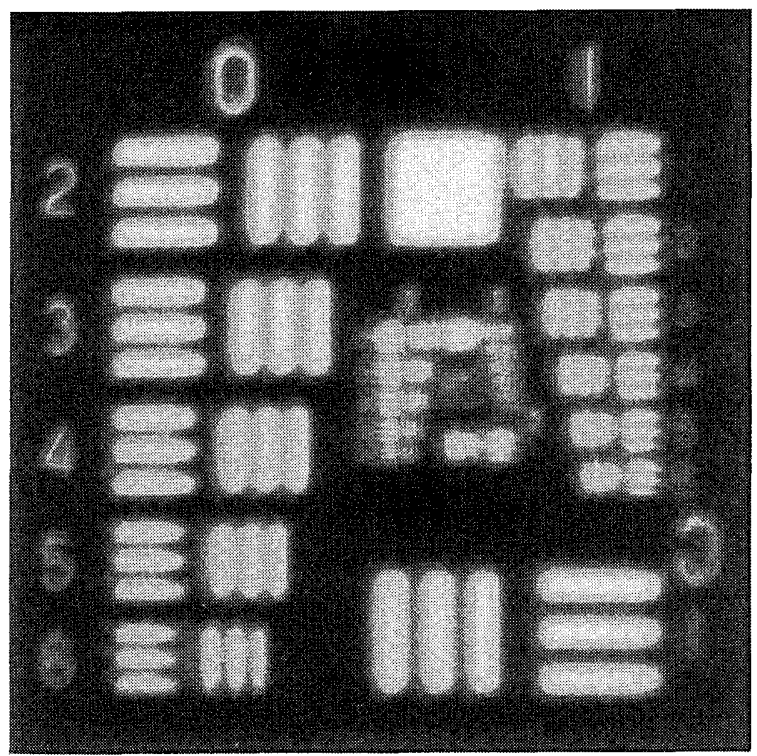

Fig. 7. Unfiltered input imaged through the inhomogeneity (distorted image).

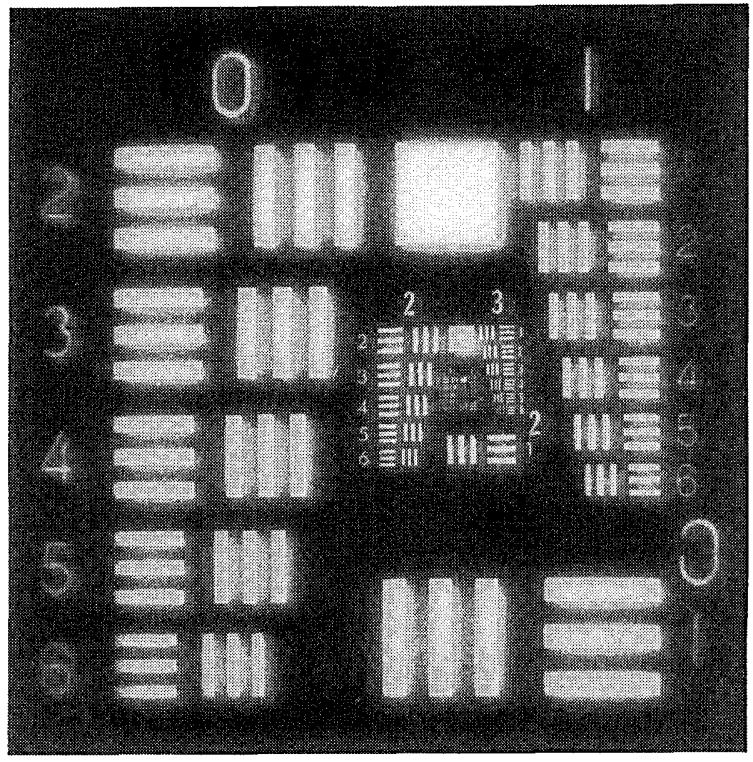

Fig. 8. Prefiltered input imaged through the inhomogeneity. The prefiltered image is insensitive to the inhomogeneity.

transmitted without the inhomogeneity (perfect image). Figure 7 shows the unfiltered input imaged through the inhomogeneity (corrupted image). Figure 8 is the filtered input imaged through the inhomogeneity. For comparison, Fig 9 shows the filtered input imaged without the inhomogeneity.

The similarity in Figs. 8 and 9 shows that the spatial filter has indeed made the imaging system insensitive to the inhomogeneity. As expected, Fig. 8 is not quite as good as the original of Fig. 6 . As discussed in the previous section, this is mostly due to the nature of the 


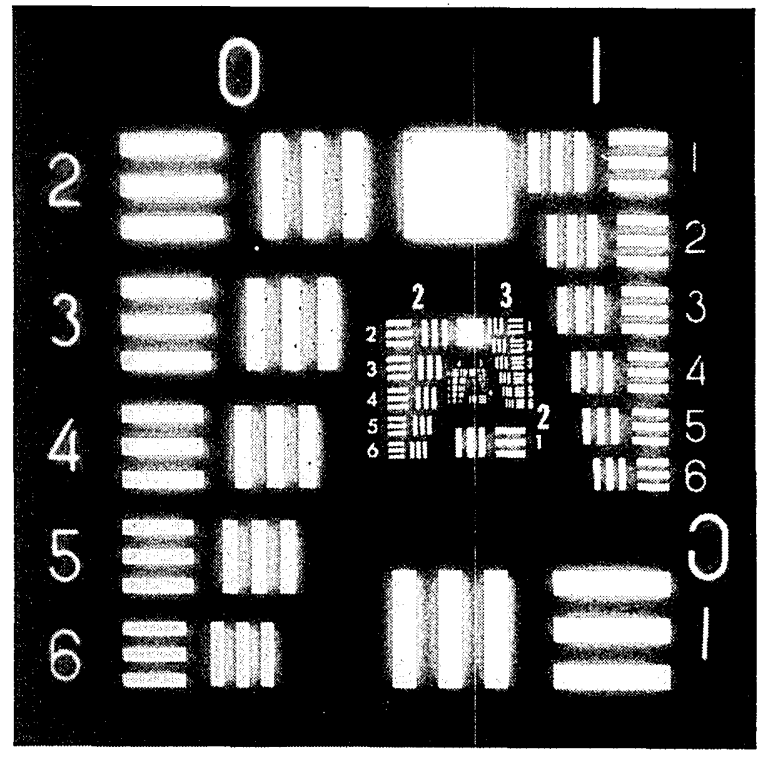

Fig. 9. Prefiltered input imaged without the inhomogeneity for comparison with Fig. 8.

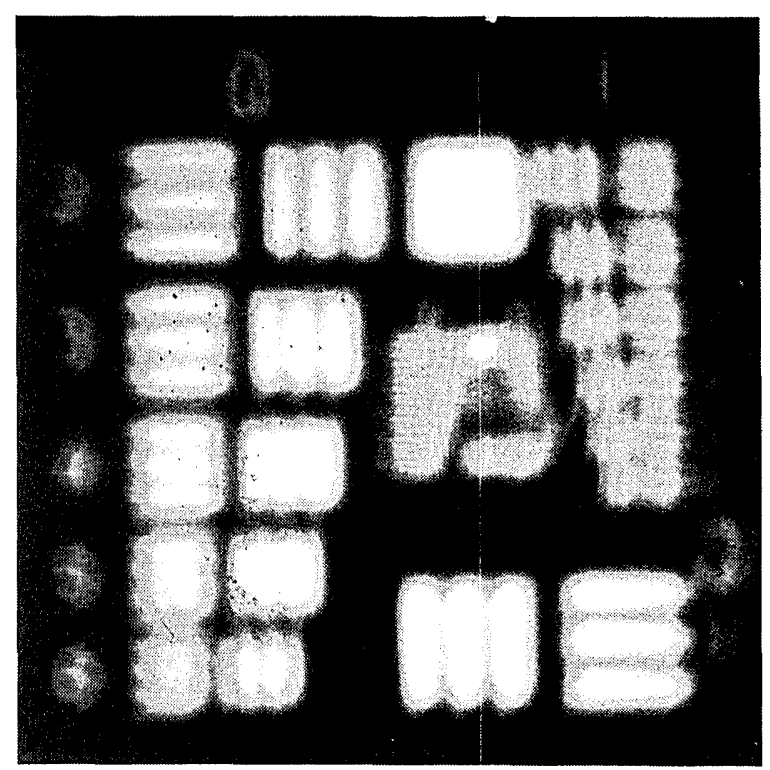

Fig. 10. Input imaged through the inhomogeneity in a direction inverse of that shown in Fig. 3.

filter, which leads to a MTF having a large autocorrelation peak and broad wings. The broad wings mean that a large band of spatial frequencies is being transmitted, but the large peak near zero frequency results in a poor contrast.

The input can also be transmitted first through the inhomogeneity and then filtered on the output side. The setup used to demonstrate this was the same as that of Fig. 4 except that the position of the input and that of the output were inverted. The results were essentially the same as those of the first experiment, as is shown in Figs. 10 and 11. Figures 10 and 11 show the

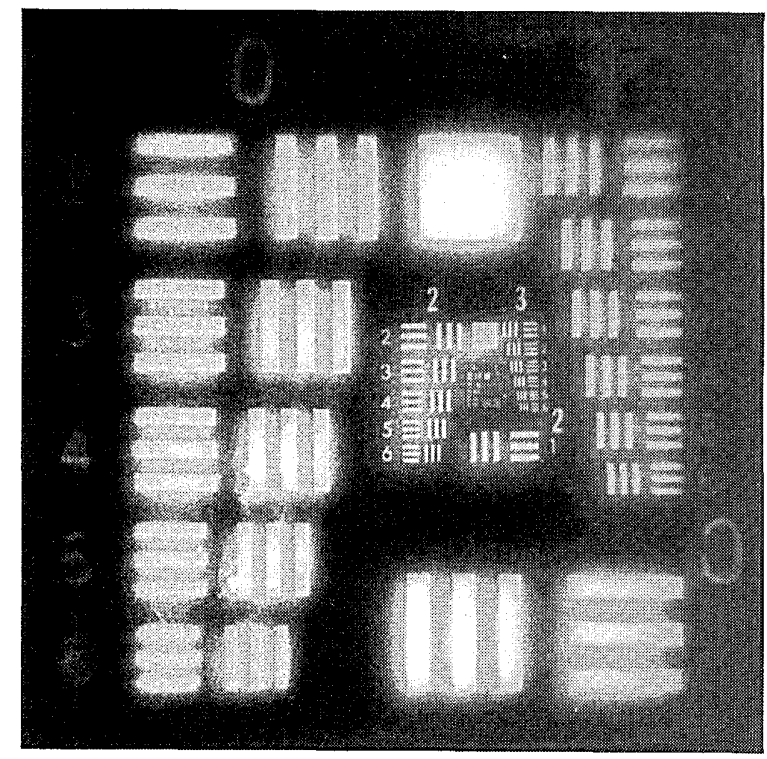

Fig. 11. Input imaged through the inhomogeneity and then postfiltered to extract $a$ posteriori the image information which was transmitted integrally through the inhomogeneity.

unfiltered and filtered outputs, respectively. In both cases, the input was first transmitted through the inhomogeneity before being filtered. The inhomogeneity was somewhat more severe than for the first experiment, leading to the more severely degraded image of Fig. 10.

In principle, the input should be illuminated with quasimonochromatic light having the same average wavelength as that used to record the filter. This requirement, however, is surprisingly weak. For the results shown, tungsten light filtered with a broadband red gelatin filter was used. No significant improvement was observed when a narrowband interference filter was used instead.

\section{v. Conclusions}

We have demonstrated that distortion free imaging through a system with aberrations that can be localized in the pupil plane is possible if the optical system selects a set of spatial frequencies which is identically affected by the inhomogeneity (i.e., experience identical phase shifts $\bmod 2 \pi$ ). Perhaps one of the main advantages of this technique is the simplicity with which the necessary spatial filter can be calculated or recorded as an interferogram. This step could in principle be realized in real time, using high speed phase shifting interferometry to acquire the data and a realtime spatial light modulator to realize the filter. Such a device would make it possible to implement an adaptive system in which the filter is continually updated to match the dynamic changes of the inhomogeneous medium.

The research leading to this paper was financially supported in part by a grant from the Thomas F. and Kate Miller Jeffress Memorial Trust. I wish to ex- 
press my appreciation to a reviewer for some very useful comments.

\section{References}

1. J. Tsujiuchi, "Restitution des images aberrantes par le filtrage des frequences spatiales," Opt. Acta 7, 243-261 (1960).

2. E. N. Leith and J. Upatnieks, "Holograms: Their Properties and Uses," SPIE J. 4, 3-6 (1965).

3. H. Kogelnik, "Holographic Image Projection Through Inhomogeneous Media," Bell Syst. Tech. J. 44, 2451-2455 (1965).

4. O. Yu Nosach, V. Il Popovichev, V. V. Ragulsky, and F. S. Faizullow, "Compensation of Phase Distortions in an Amplifying Medium by a Brillouin Mirror," JETP Lett. 16, 435-437 (1972).

5. R. W. Hellwarth, "Generation of Time Reversed Wavefronts by Nonlinear Reflection," J. Opt. Soc. Am. 67, 1-3 (1977).

6. R. Risher, Ed., Optical Phase Conjugation (Academic, New York, 1983).

7. J. Munch, R. Wuerker, and L. Heflinger, "Wideband Holographic Correction of an Aberrated Telescope Objective," Appl. Opt. 29, 2440-2445 (1990).
8. J. Upatnieks, A. Vander Lugt, and E. Leith, "Correction of Lens Aberrations by Means of Holograms," Appl. Opt. 5, 589-593 (1966).

9. A. Yariv and T. L. Koch, "One-Way Coherent Imaging Through a Distorting Medium Using Four-Wave Mixing," Opt. Lett. 7, 113-115 (1982).

10. K. R. MacDonald, W. R. Tompkin, and R. W. Boyd, "Passive One-Way Aberration Correction Using Four-Wave Mixing," Opt. Lett. 13, 485-487 (1988).

11. E. N. Leith, D. K. Angell, and C.-P. Kuei, "Superresolution by Incoherent-to-Coherent Conversion," J. Opt. Soc. Am. A 4, 1050-1054 (1987).

12. A. Cunha and E. N. Leith, "Generalized One-Way Phase-Conjugation Systems," J. Opt. Soc. Am. B 6, 1803-1812 (1989).

13. F. Merkle, "New Adaptive Optics Results from ESO," in ICO 15, Garmish-Partenkirchen (1990), postdeadline paper; see also "Successful Test of Adaptive Optics," EO Reports 75, 1 (Mar. 1990).

14. J. W. Goodman, Introduction to Fourier Optics (McGraw-Hill, San Francisco, 1968), Chap. 6. 